\title{
Short- and long-term efficacy of negative-pressure wound therapy in split-thickness skin grafts: a retrospective study
}

\author{
Ran Mo ${ }^{1,2 \#}$, Zhouji Ma ${ }^{1 \#}$, Chen Chen ${ }^{3 \#}$, Xueyong Meng', Qian Tan ${ }^{1,2}$ \\ ${ }^{1}$ Department of Burns \& Plastic Surgery, Nanjing Drum Tower Hospital Clinical College of Nanjing Medical University, Nanjing, China; \\ ${ }^{2}$ Department of Burns \& Plastic Surgery, Nanjing Drum Tower Hospital, the Affiliated Hospital of Medical School of Nanjing University, Nanjing, \\ China; ${ }^{3}$ Department of Nutrition, Nanjing Drum Tower Hospital, the Affiliated Hospital of Medical School of Nanjing University, Nanjing, China \\ Contributions: (I) Conception and design: R Mo; (II) Administrative support: Q Tan; (III) Provision of study materials or patients: Z Ma; (IV) \\ Collection and assembly of data: Z Ma, C Chen; (V) Data analysis and interpretation: R Mo, Z Ma; (VI) Manuscript writing: All authors; (VII) Final \\ approval of manuscript: All authors. \\ \#These authors contributed equally to this work. \\ Correspondence to: Qian Tan. Department of Burns \& Plastic Surgery, Nanjing Drum Tower Hospital Clinical College of Nanjing Medical University; \\ Department of Burns \& Plastic Surgery, Nanjing Drum Tower Hospital, the Affiliated Hospital of Medical School of Nanjing University, 321 \\ Zhongshan Road, Nanjing 210008, China. Email: smmutanqian@sina.com.
}

Background: Graft fixation is essential for the successful survival of skin grafts. Negative-pressure wound therapy (NPWT) can be utilized for fixing a skin graft, ensuring adhesion of the graft with continuous and uniform pressure. However, the reported short- and long-term efficacy of NPWT in split-thickness skin grafts (STSGs) is inconsistent, with few studies on the long-term efficacy (scar quality). To clarify the appropriate methods of skin graft fixation, we conducted a single-center retrospective study on the shortand long-term effects of skin grafting using different fixation methods.

Methods: This study retrospectively analyzed patients who underwent STSG from December 2010 to June 2019. The patients were divided into two groups based on the skin graft-fixing method: an NPWT group and a conventional mechanical fixation group. Medical data including age, sex, underlying diseases, wound etiology, recipient site, surgical methods, surgical outcomes, postoperative complications, and follow-up data (Vancouver Scar Scale score and Patient and Observer Scar Assessment Scale score) were analyzed.

Results: A total of 392 cases were ultimately included in the analysis. Among them, 218 cases were fixed with NPWT for skin grafting and 174 with conventional mechanical fixation. No significant differences in baseline data were noted between the two groups. The total graft survival rate in the NPWT group was higher than that in the conventional mechanical fixation group (86.7\% vs. $74.1 \%, \mathrm{P}=0.002)$. Moreover, the infection rate in the NPWT group was lower than that in the conventional mechanical fixation group (5.5\% vs. $13.2 \%, \mathrm{P}=0.008)$. In terms of scar quality, no significant difference was observed, except for in the hand. Overall, the scar surface regularity was better in the NPWT group than in the control group. ( $\mathrm{P}=0.019$ for Patient Scar Assessment Scale, P=0.025 for Observer Scar Assessment Scale).

Conclusions: NPWT is an effective approach for fixing skin grafts. Compared with conventional mechanical fixation, NPWT can significantly improve the survival rate and reduce the infection rate of STSG. In the long-term, NPWT can also improve scar surface regularity in the hand, with an esthetic effect that is more satisfactory to clinicians and patients.

Keywords: Negative-pressure wound therapy (NPWT); skin graft; survival rate; scar

Submitted Sep 08, 2020. Accepted for publication Dec 17, 2020.

doi: 10.21037/apm-20-1806

View this article at: http://dx.doi.org/10.21037/apm-20-1806 


\section{Introduction}

Skin grafts have been widely used for skin reconstruction due to burns, trauma, or iatrogenic defects (1), and graft fixation is essential for successful graft survival (2). Traditionally, skin grafts are secured with elastic dressings or tie-over bolsters to obtain sufficient adhesion to the wound bed (3). Improper skin graft fixation may lead to hematoma, infection, dislocation, or graft loss, significantly reducing graft survival (4). Negative-pressure wound therapy (NPWT), also known as vacuum-assisted closure (VAC), was initially introduced for suction therapy of various wounds. NPWT has been used as a dressing for patients with open fractures and acute or chronic burn wounds in an effort to reduce the extent of the injury (5). Postoperative use of VAC can create a controlled and closed environment for continuous drainage of blood and exudates, thereby promoting local circulation and tissue granulation (6). NPWT can be used as a bridge before skin transplantation to improve the quality of the wound bed and may also be applied as a dressing for the donor site of a graft. Moreover, there is a report of the application of NPWT for fixing the graft, ensuring adhesion of the skin graft with continuous and uniform pressure, and the firm fixation of a graft on the surface of the complex wound (7). Nonetheless, some randomized controlled trials have shown that the effect of NPWT on skin grafts is not superior to that of traditional dressings $(8,9)$.

In addition, NPWT or incisional NPWT (iNPWT) has been found to improve the quality of scars during wound healing (10). Indeed, according to a study on acute burns of the hand, satisfactory scar appearance and quality were obtained by using local NPWT (11). Conversely, NPWT was reported to lead to worse scar appearance when applied for open abdominal wounds (12). Overall, research on the quality of scars after skin grafts are fixed by NPWT is limited, and the effect is not clear. Regardless, a randomized controlled trial showed that NPWT significantly improved the appearance of split-thickness skin grafts (STSGs) two weeks after surgery compared with standard packaged dressings (13), and another randomized controlled trial found that NPWT improved the scar color of STSGs (14). In contrast, the results of other randomized controlled trials have indicated that NPWT did not improve the quality or esthetic appearance of scars in STSGs compared with traditional dressings (15).

At present, the results of studies on the efficacy of NPWT for skin grafts are not consistent, especially regarding long-term effects, namely, the quality and appearance of scars. To clarify appropriate methods of skin graft fixation, we conducted a single-center retrospective study on the short- and long-term effects of skin grafting with different fixation methods. We present the following article in accordance with the STROBE reporting checklist (available at http://dx.doi.org/10.21037/apm-20-1806).

\section{Methods}

\section{Patients}

This study retrospectively analyzed patients who underwent STSG in the Department of Burns and Plastic Surgery of Nanjing Drum Tower Hospital from December 2010 to June 2019. The inclusion criteria and exclusion criteria are provided in Table 1, and the screening and exclusion process of patients is depicted in Figure 1. We excluded patients with scar release or excision and then skin grafting because sites that have formed scar contractures tend to produce bad scars, leading to bias in the evaluation of follow-up results. Written informed consent was obtained from the patient for publication of this study and any accompanying images. The study was conducted in accordance with the Declaration of Helsinki (as revised in 2013).

Ultimately, 392 of 595 patients were included in the analysis. The research scheme was approved by the Ethics Committee of Nanjing Drum Tower Hospital (2020-10901). Patient data were collected from the medical records of Nanjing Drum Tower Hospital and included age, sex, underlying diseases, wound etiology, recipient site, surgical methods, surgical outcomes, postoperative complications, and follow-up data. The patient's baseline data are shown in Table 2. The patients were divided into two groups based on the method of fixing skin graft used: an NPWT group and a conventional mechanical fixation group.

\section{Surgical methods}

STSGs were harvested from the scalp, abdomen, or thigh with a pneumatic-driven dermatome (Zimmer Biomet Air Dermatome; Zimmer Biomet, Warsaw, IN, USA). The thickness of the STSGs ranged from 0.02 to $0.03 \mathrm{~cm}$. The graft was perforated with an 11-blade scalpel and then affixed to the wound for good drainage. After preparing the wound (thorough debridement, saline wash, and rinsing with antibiotic fluids) (Figure 2A), a skin graft was applied and secured with intermittent nonabsorbable sutures as 
Table 1 Inclusion and exclusion criteria

Inclusion criteria

- Patients presenting with a wound that requires a split-thickness skin graft

- Graft fixation with NPWT or conventional mechanical fixation

- Patients aged 18 to 80 years

- Operation performed by three senior surgeons

Exclusion criteria

- Patients with dermatological skin diseases at the edge of the wound

- Patients with underlying diseases such as SLE, hepatic failure, HIV/AIDS

- Patients using immunosuppressive or chemotherapeutic agents, or those undergoing radiation therapy

- Patients who were allergic to or had a hypersensitivity to the dressing material

- Patients who underwent skin grafts for scar release or excision

- Lost to follow-up

NPWT, negative pressure wound therapy; SLE, systemic lupus erythematosus; HIV, human immunodeficiency virus; AIDS, acquired immunodeficiency syndrome.

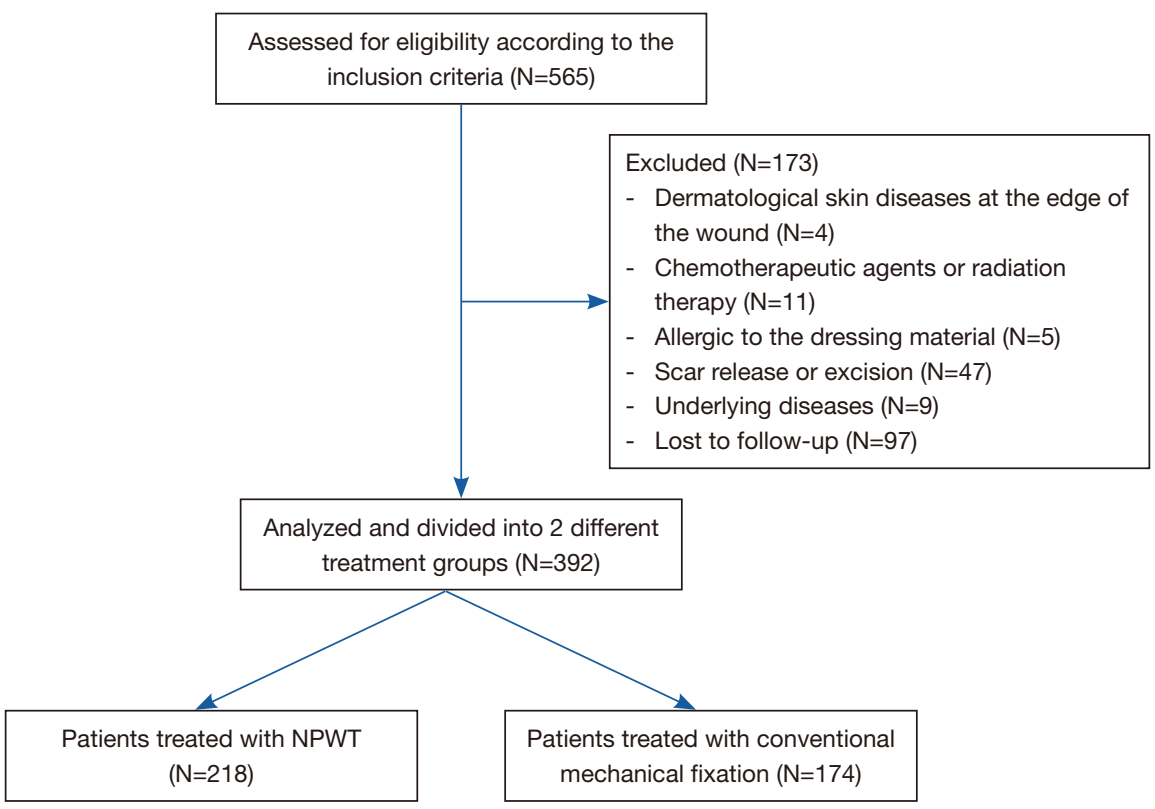

Figure 1 Flowchart of patient selection algorithm based on inclusion and exclusion criteria (Table 1). NPWT, negative-pressure wound therapy.

needed (Figure 2B). Washing with saline was repeated, and the grafts were covered with nonadhesive petroleum jelly gauze. The skin grafts were fixed with either a conventional mechanical dressing or NPWT.

In the conventional mechanical fixation group, the graft was laid over the entire surface of the wound bed without stretching or wrinkling. First, the graft was sutured using 3-0 or 4-0 nylon anchoring sutures, depending on the surface of the wound bed. The distance between each suture was 4-10 mm. Subsequently, petrolatum gauze covered with cotton balls or loose gauze was tied over the sutures with the aid of an assistant who held them in place to prevent slippage. 
Table 2 Baseline data of both groups

\begin{tabular}{|c|c|c|c|c|}
\hline Characteristics & Total $(\mathrm{N}=392)$ & NPWT (N=218) & Convention (N=174) & $\mathrm{P}$ \\
\hline Gender (male vs. female) & $275: 117$ & $161: 57$ & $114: 60$ & 0.073 \\
\hline Hypertension & $36(9.2 \%)$ & $19(8.7 \%)$ & 17 (9.8\%) & 0.719 \\
\hline Diabetes & $29(7.4 \%)$ & $18(8.3 \%)$ & $11(6.3 \%)$ & 0.467 \\
\hline Burn injury & $115(29.3 \%)$ & $64(29.4 \%)$ & $51(29.3 \%)$ & \\
\hline Traumatic wound & $98(25.0 \%)$ & $55(25.2 \%)$ & $43(24.7 \%)$ & \\
\hline Tumor resection & 139 (35.5\%) & $73(33.5 \%)$ & $66(37.9 \%)$ & \\
\hline Others & $40(10.2 \%)$ & $26(11.9 \%)$ & $14(8.0 \%)$ & \\
\hline Hand & $67(17.1 \%)$ & $28(11.9 \%)$ & $39(22.4 \%)$ & \\
\hline Foot & $67(17.1 \%)$ & $36(16.5 \%)$ & $31(17.8 \%)$ & \\
\hline Upper extremities & $36(9.2 \%)$ & $20(9.2 \%)$ & $16(9.2 \%)$ & \\
\hline Lower extremities & 115 (29.3\%) & $70(32.1 \%)$ & $45(25.9 \%)$ & \\
\hline Trunk & $44(11.2 \%)$ & $28(12.8 \%)$ & $16(9.2 \%)$ & \\
\hline
\end{tabular}

NPWT, negative pressure wound therapy. Others: donor site of flap surgery, Postoperative incision nonunion, and soft tissue infection.

The outermost layer was wrapped with aseptic dressing and elastic bandage. Four or five days after the surgery, the tieover was removed at the bedside, and the site was examined. This technique has been widely employed for graft fixation because it ensures contact between the graft and the wound bed, thereby preventing hematoma accumulation (16).

In the NPWT group, a sponge (GRANUFOAM Dressing; KCI, an Acelity Company, San Antonio, TX, USA) was trimmed to an appropriate contour to cover the grafted area; a suture or nail was applied when needed to cover an extensive surface. Adhesive laminate was applied over the sponge and surrounding the intact skin. Then, the sponge was connected to a portable unit after the seal coverage was secured (Figure 2C). The vacuum mode was set at continuous $-75 \mathrm{mmHg}$ (Figure $2 D$ ). No central negative pressure was used under any circumstances. The NPWT unit automatically detects leakage of the sponge, with higher indicating stripes indicating more leakage. The machine sounds an alarm when the instruction strip exceeds the warning line. Loss of vacuum pressure was controlled by adding laminated film dressings. The sponges on the skin were removed at 5-7 days after the operation. After the
NPWT sponge was removed, the graft was covered with petroleum jelly gauze and fixed with appropriate routine fixation (once the NPWT sponge was removed, the graft was fixed with a dressing). Fixing materials included gauze, elastic and inelastic bandages and splints (if necessary). Care was taken to ensure that pressure did not impair the blood supply of the graft.

\section{Outcome evaluation}

The results of the STSG were analyzed and recorded by two attending doctors after the operation. The evaluation included total graft survival, partial graft failure, complete graft failure, hematoma formation, and graft infection. Total graft survival is healed by initial complete epithelialization. Total graft failure usually required additional skin grafting. (The fixation method was the same as before). Partial graft failure meant that a small part of the graft was lost, but it could heal itself through a change of dressing. Graft survival was evaluated 10-14 days after the operation. The formation of hematoma could be observed when the dressing was opened. The infection was judged by the two 

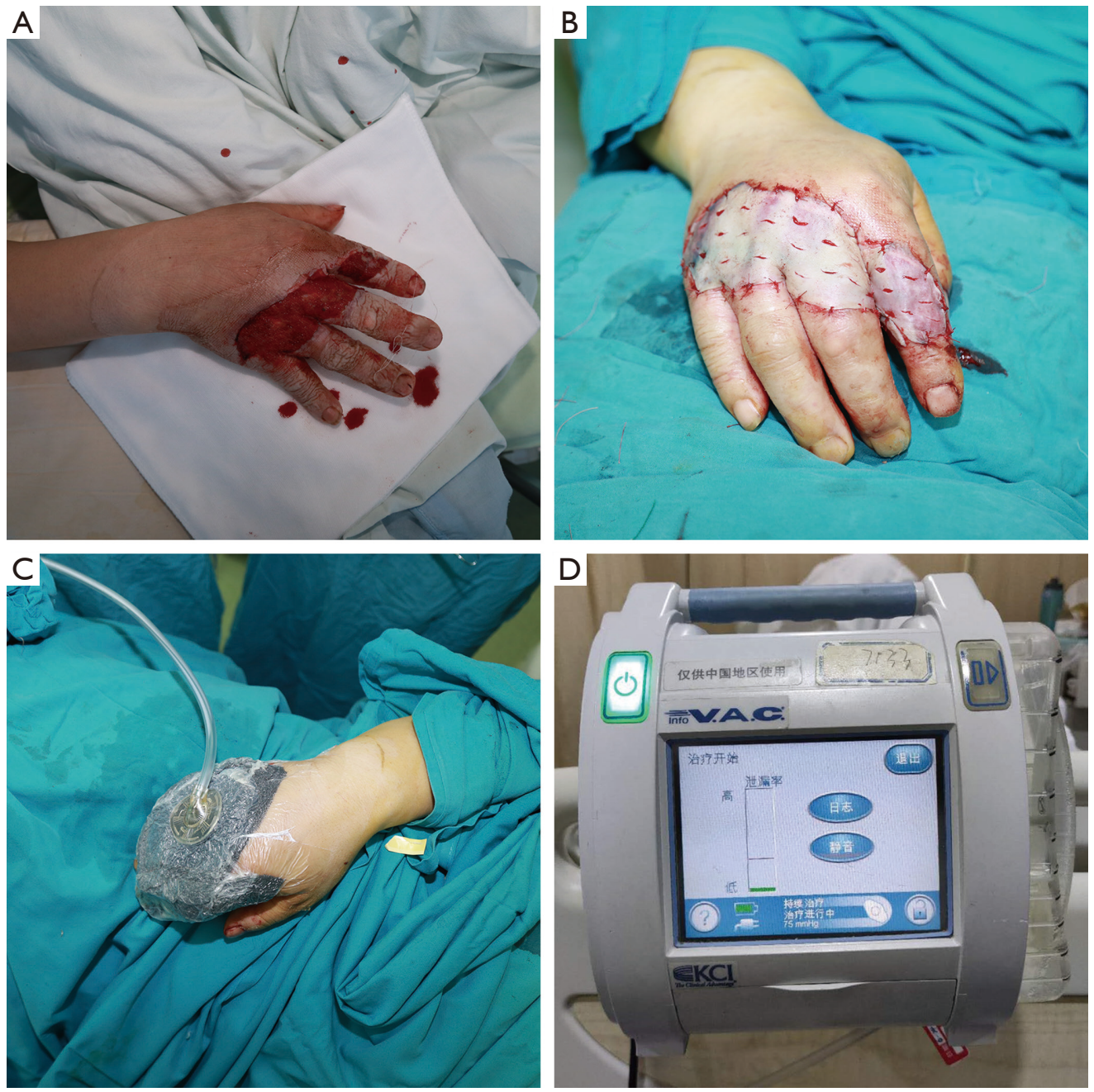

Figure 2 Surgical procedure and NPWT application. (A) Preoperatively; (B) detect covered with a split-thickness skin graft; (C) the splitthickness fixed with NPWT; (D) The vacuum mode of portable unit was set at continuous $-75 \mathrm{mmHg}$. The NPWT unit automatically detects leakage of the sponge, with higher indicating stripes indicating more leakage. The machine sounds an alarm when the instruction strip exceeds the warning line. NPWT, negative-pressure wound therapy.

attending physicians according to the clinical characteristics and microbiological results of the wound when the dressing was opened.

\section{Follow-up}

Follow-up was carried out by telephone, outpatient clinic visits, or online communication software. Patients were followed up every three months after discharge from the hospital, and the total follow-up duration was more than 1 year. The Vancouver Scar Scale (VSS) and Patients and Observers Scar Assessment Scale (POSAS) were applied to assess scar quality during follow-up. The data for the 1-year follow-up were included in analysis.

\section{VSS}

The VSS is a widely used tool for evaluating scar quality. This scale is a clinical evaluation of four variables, namely, "vascularization" $(0$ points $=$ normal color, 1 point $=$ pink, 2 points = dark red, or 3 points = purple $)$, "height" $(0$ points = flat, 1 point $=$ less than $2 \mathrm{~mm}, 2$ points $=2-5 \mathrm{~mm}$, 3 points $=$ more than $5 \mathrm{~mm})$, "flexibility" $(0=$ normal, $1=$ soft, $2=$ yielding, $3=$ hard, $4=$ ropelike, or $5=$ contracture) 
and "pigmentation" $(0=$ normal, $1=$ hypopigmentation, $2=$ mixed, $3=$ hyperpigmented). The height of the scar was measured with a simple measuring stick, and the flexibility of the scar is evaluated by creating skin folds. Each variable had four to six levels, as specified above. Total possible scores ranged from 0 to 14 , with a score of 0 indicating normal skin.

\section{Patient and Observer Scar Assessment Scale (POSAS)}

The POSAS consists of two scales: the OSAS and Patient Scar Assessment Scale (PSAS). All items of the two scales are scored numerically. Five parameters, namely, vascularization, pigmentation, pliability, thickness, and relief (the extent to which surface irregularities are present), were scored by the observer. Six parameters, including pain, itching, color, stiffness, thickness, and irregularity, were scored by the patients. Each item has a 10-step score, whereby a score of 10 reflects the worst imaginable scar, sensation, or evaluation. In addition, the observers and patients were asked to offer an overall opinion, also on a scale of 1 to 10 , but not included in the total score. The total score ranged from 5 to 50 for the observer scale of the POSAS and from 6 to 60 for the patient score of the POSAS. POSA has three more items than VSS, pain, itching, and relief, and takes into account the subjective opinions of patients and observers.

\section{Statistical analysis}

Statistical analysis and graphing were performed using IBM SPSS version 26.0 (SPSS, Chicago, IL, USA, RRID: SCR_002865) and GraphPad version 8.0 (GraphPad Software, San Diego, CA, USA, RRID: SCR_002798). The normality of the distribution was tested by calculating skewness and kurtosis, evaluating a frequency histogram, and performing the Shapiro-Wilk test. An independent $t$-test (in the case of a normal distribution) or MannWhitney $U$ test (in the case of a nonnormal distribution) was used to test for significant differences between data from independent groups. To assess the differences in nonlevel count data, a chi-square test, continuity correction, or Fisher exact test was used. The two-tailed significance threshold was set at 0.05 .

\section{Results}

Among the patients who underwent STSG from December
2010 to June 2019, 565 were initially enrolled; 173 patients were excluded according to the exclusion criteria, and thus 392 patients were ultimately included in the analysis. Among them, 218 (male, 161; female, 57) and 174 (male, 114; female, 60) cases were fixed with NPWT and conventional mechanical fixation for skin grafting. The average age of the NPWT group was $50.45 \pm 16.48$ years, and that of the conventional mechanical fixation group was $47.66 \pm 16.88$ years. Patients with high blood pressure and diabetes were included in this study. The wound etiology involved burn injury, traumatic wound, resection of skin tumor, donor area of a flap, nonhealing of postoperative incision, and soft tissue infection. The recipient site included the head and neck, hands, foot, upper extremities, lower extremities, and trunk. The baseline information and clinical features of both groups are provided in Table 2. There was no significant difference in age, sex, underlying diseases, wound etiology, or recipient site between the two groups.

The results and complications of all skin grafts are summarized in Table 3. The total graft survival rate in the NPWT group $(86.7 \%)$ was higher than that in the conventional mechanical fixation group $(74.1 \%)(\mathrm{P}=0.002)$. With regard to each recipient site, the total graft survival rates in the NPWT group for head and neck $(88.7 \%$ vs. $74.1 \%, \mathrm{P}=0.031)$, hand $(89.3 \%$ vs. $66.7 \%, \mathrm{P}=0.032)$ and foot $(88.7 \%$ vs. $67.8 \%, \mathrm{P}=0.034)$ were higher than those in the control group. However, there was no statistical significance for the upper extremities $(90.0 \%$ vs. $81.3 \%$, $\mathrm{P}=0.778)$, lower extremities $(82.9 \%$ vs. $82.2 \%, \mathrm{P}=0.930)$ or trunk $(85.7 \%$ vs. $87.5 \%, \mathrm{P}=0.868)$ (Figure 3). Moreover, the total graft failure, that is, the rate of reoperation, was lower in the NPWT group than in the conventional mechanical fixation group ( $2.8 \%$ vs. $5.2 \%, \mathrm{P}=0.215)$; however, there was no statistical significance, and there was no difference for each recipient site. Additionally, no significant difference in the incidence of hematoma between the two groups ( $5.5 \%$ vs. $7.5 \%, \mathrm{P}=0.429$ ) or for each site was detected. The infection rate in the NPWT group was lower than that in the conventional mechanical fixation group $(5.5 \%$ vs. $13.2 \%$ $\mathrm{P}=0.008)$. Despite a significant difference in the foot $(2.8 \%$ vs. $22.6 \%, \mathrm{P}=0.034)$ and lower extremities $(5.7 \%$ vs. $20.0 \%$, $\mathrm{P}=0.018)$, there was no statistical significance observed for the other recipient sites (Figure 4).

In terms of long-term scar quality, VSS scores are shown in Table 4. The total score of the NPWT group was lower than that of the conventional mechanical fixation group, but with no significant difference $(\mathrm{P}=0.465)$. Moreover, there was no significant difference among each subitem with 
Table 3 Recipient sites and outcomes of skin grafts

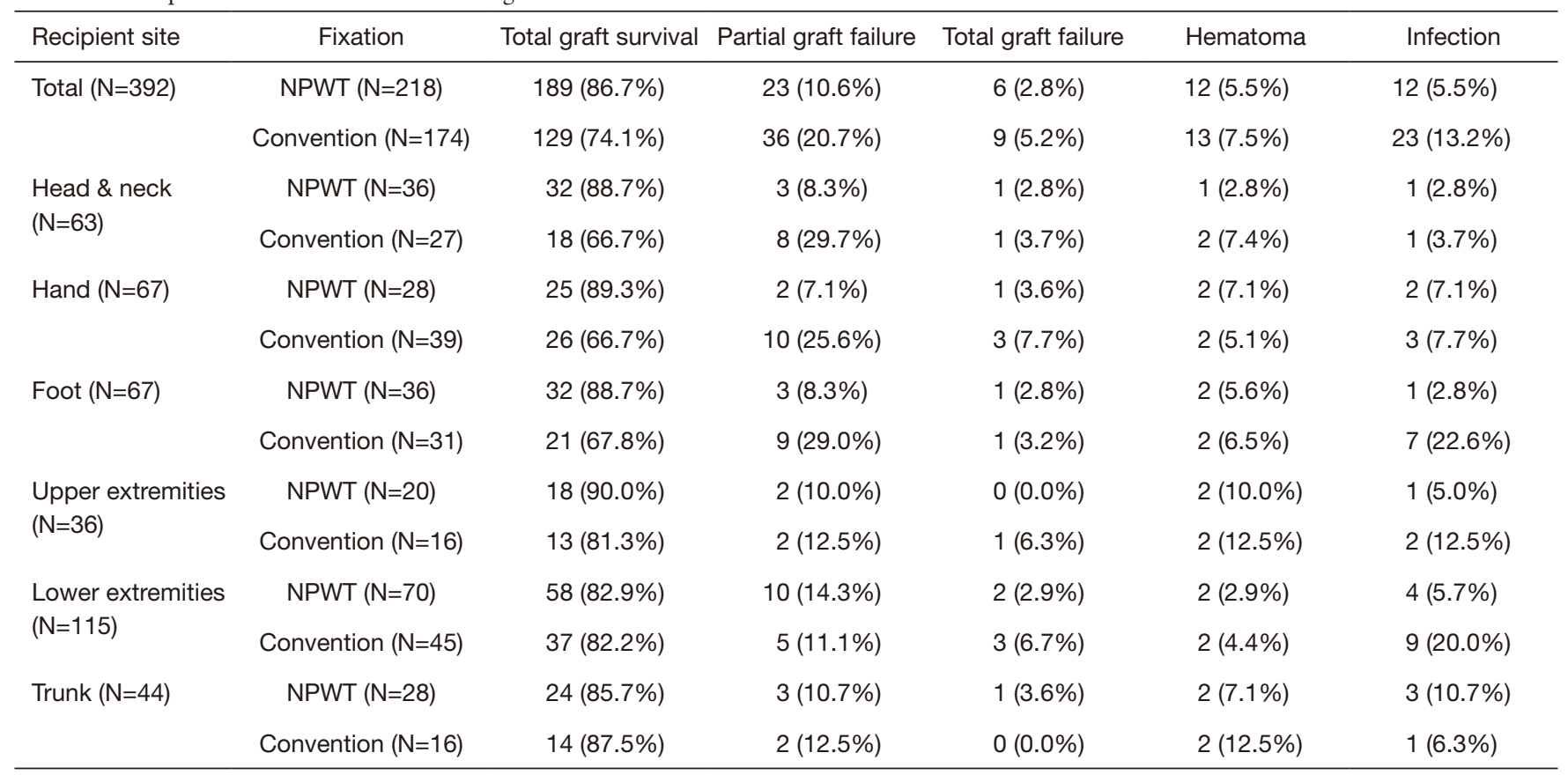

NPWT, negative pressure wound therapy.

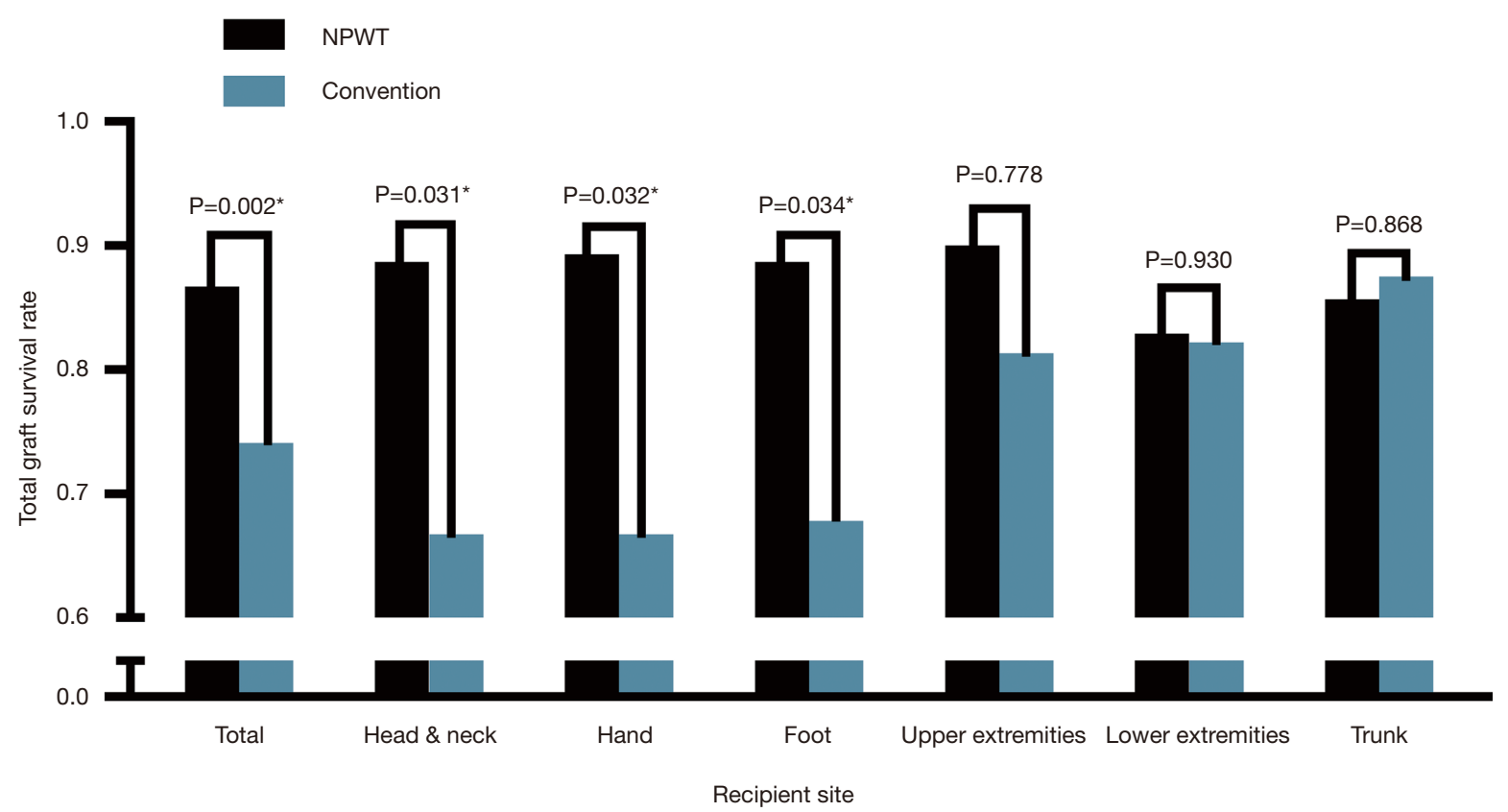

Figure 3 Total graft survival rate outcomes with NPWT or conventional mechanical fixation. *, $\mathrm{P}<0.05$. NPWT, negative-pressure wound therapy. 


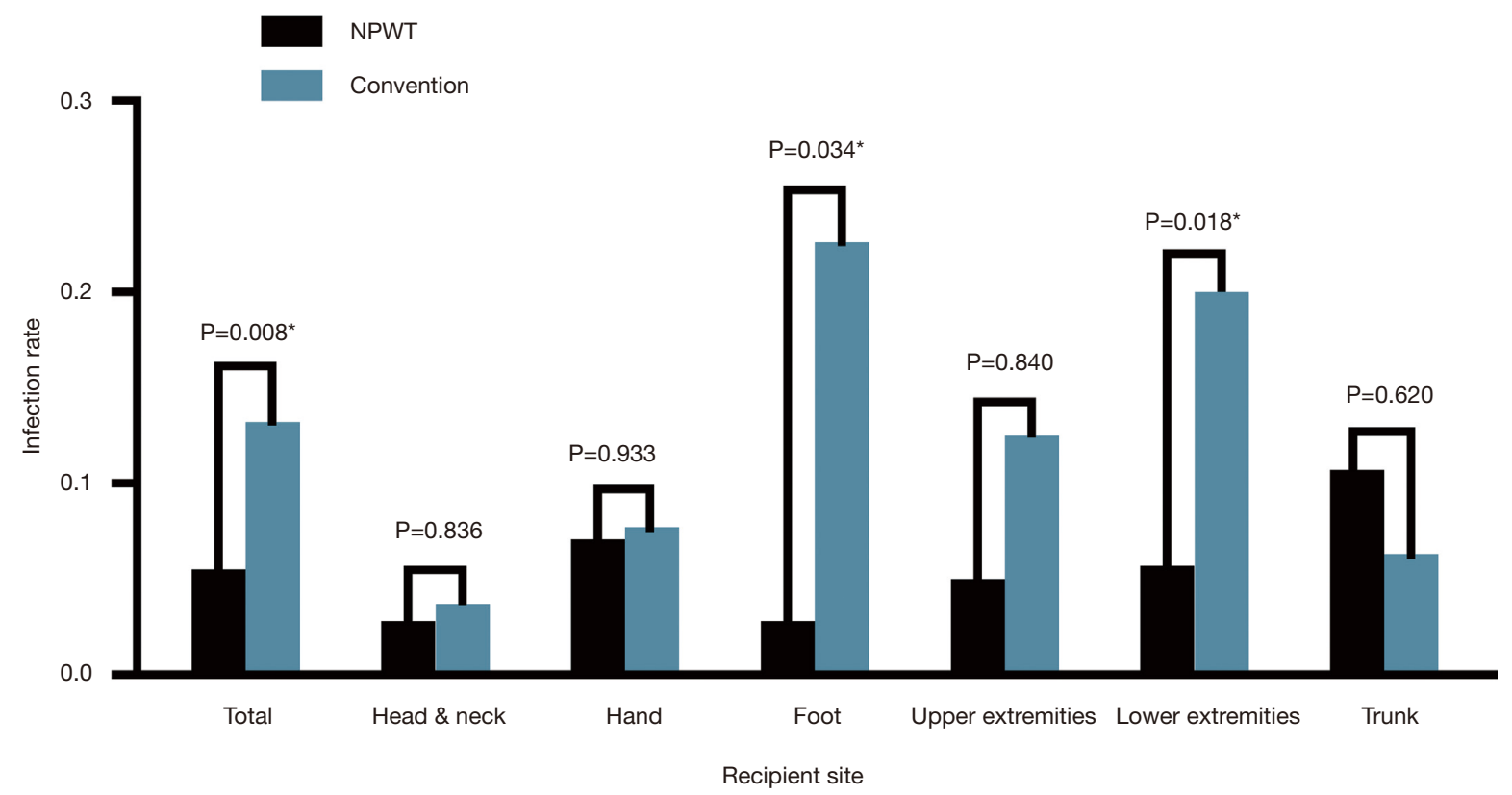

Figure 4 Infection rate outcomes with NPWT or conventional mechanical fixation. *, $\mathrm{P}<0.05$. NPWT, negative-pressure wound therapy.

Table 4 VSS score of both groups

\begin{tabular}{lccc}
\hline VSS & NPWT & Convention & $P$ \\
\hline Pigmentation & $1.89 \pm 0.62$ & $1.59 \pm 0.59$ & 0.609 \\
Vascularity & $1.85 \pm 0.64$ & $1.86 \pm 0.62$ & 0.862 \\
Pliability & $2.05 \pm 0.78$ & $2.07 \pm 0.81$ & 0.872 \\
Height & $1.79 \pm 0.56$ & $1.82 \pm 0.55$ & 0.614 \\
Total & $7.56 \pm 1.28$ & $7.65 \pm 1.36$ & 0.465 \\
\hline
\end{tabular}

VSS, Vancouver scar scale; NPWT, negative pressure wound therapy.

regard to pigmentation $(\mathrm{P}=0.609)$, vascularization $(\mathrm{P}=0.862)$, pliability $(\mathrm{P}=0.872)$ and height $(\mathrm{P}=0.614)$. In the PSAS, the total score $(\mathrm{P}=0.243)$ and the overall opinion $(\mathrm{P}=0.393)$ of the NPWT group were better, but no significant difference between the two groups was observed. For the OSAS, the total score $(\mathrm{P}=0.380)$ and the overall opinion $(\mathrm{P}=0.273)$ of the NPWT group were also better, but again with no significant difference. No significant difference was found in each POSA subitem (Table 5).

In further analysis of scar quality for each specific anatomical location, we observed no significant difference, except for the hand. In the PSAS score of the hand, the scar surface regularity of the NPWT group was better than that of the conventional mechanical fixation group
Table 5 POSAS score of both groups

\begin{tabular}{lccc}
\hline Item & NPWT & Convention & $\mathrm{P}$ \\
\hline PSAS & & & \\
Pain & $2.33 \pm 1.16$ & $2.32 \pm 1.18$ & 0.911 \\
Itch & $2.19 \pm 1.09$ & $2.20 \pm 0.97$ & 0.539 \\
Color & $3.70 \pm 1.33$ & $3.78 \pm 1.34$ & 0.639 \\
Stiffness & $3.95 \pm 1.57$ & $4.11 \pm 1.65$ & 0.551 \\
Thickness & $3.62 \pm 1.23$ & $3.67 \pm 1.22$ & 0.620 \\
Irregularity & $2.79 \pm 1.04$ & $2.90 \pm 1.15$ & 0.349 \\
Total & $18.58 \pm 3.42$ & $18.98 \pm 3.23$ & 0.243 \\
Overall opinion & $3.22 \pm 0.67$ & $3.29 \pm 0.60$ & 0.393 \\
OSAS & & & \\
Vascularity & $3.70 \pm 1.33$ & $3.70 \pm 1.25$ & 0.978 \\
Pigmentation & $3.79 \pm 1.33$ & $3.83 \pm 1.32$ & 0.995 \\
Thickness & $3.61 \pm 1.25$ & $3.66 \pm 1.22$ & 0.629 \\
Relief & $2.80 \pm 1.03$ & $2.89 \pm 1.10$ & 0.499 \\
Pliability & $4.00 \pm 1.55$ & $4.12 \pm 1.61$ & 0.739 \\
Total & $17.89 \pm 3.28$ & $18.19 \pm 3.13$ & 0.380 \\
Overall opinion & $3.47 \pm 1.01$ & $3.60 \pm 0.92$ & 0.273 \\
\hline POSAS, patient & $a n d 0 b s e r v e r$ & $56 a r a s 5959$ \\
\hline
\end{tabular}

POSAS, patient and observer scar assessment scale; PASA, patient scar assessment scale; OSAS, observer scar assessment scale; NPWT, negative pressure wound therapy. 
Table 6 POSAS score of hands in both groups

\begin{tabular}{lccc}
\hline Item & NPWT & Convention & $\mathrm{P}$ \\
\hline PSAS & & & \\
Pain & $1.75 \pm 1.08$ & $1.97 \pm 1.16$ & 0.292 \\
Itch & $2.04 \pm 1.26$ & $2.18 \pm 0.97$ & 0.230 \\
Color & $3.57 \pm 1.29$ & $3.77 \pm 1.38$ & 0.547 \\
Stiffness & $3.89 \pm 1.57$ & $4.08 \pm 1.71$ & 0.644 \\
Thickness & $3.39 \pm 1.28$ & $3.51 \pm 1.15$ & 0.595 \\
Irregularity & $2.71 \pm 1.18$ & $3.38 \pm 1.25$ & $0.019^{\star}$ \\
Total & $17.36 \pm 3.69$ & $18.92 \pm 3.11$ & 0.065 \\
Overall opinion & $2.68 \pm 0.82$ & $3.18 \pm 0.68$ & $0.013^{\star}$ \\
OSAS & & & \\
Vascularity & $3.75 \pm 1.38$ & $3.82 \pm 1.23$ & 0.693 \\
Pigmentation & $3.64 \pm 1.22$ & $3.79 \pm 1.24$ & 0.669 \\
Thickness & $3.39 \pm 1.29$ & $3.54 \pm 1.14$ & 0.595 \\
Relief & $2.79 \pm 1.13$ & $3.36 \pm 1.18$ & $0.025^{\star}$ \\
Pliability & $3.93 \pm 1.56$ & $4.10 \pm 1.57$ & 0.630 \\
Total & $17.50 \pm 3.43$ & $18.62 \pm 3.31$ & 0.185 \\
Overall opinion & $3.18 \pm 1.06$ & $3.72 \pm 0.80$ & $0.043^{\star}$ \\
\hline & & & \\
\hline
\end{tabular}

${ }^{*}, \mathrm{P}<0.05$. POSAS, patient and observer scar assessment scale; PASA, patient scar assessment scale; OSAS, observer scar assessment scale; NPWT, negative pressure wound therapy.

based on the PSAS score of the hand. In addition, the overall opinion of the patient was better $(\mathrm{P}=0.013)$, though there was no significant difference in the total score $(\mathrm{P}=0.065)$. Regarding the OSAS score of the hand, the scar relief of the NPWT group was better than that of the conventional mechanical fixation group $(\mathrm{P}=0.025)$, and the overall opinion of the patient was better $(\mathrm{P}=0.043)$, but with no significant difference in the total score $(\mathrm{P}=0.185)$ (Table 6). Figure 5 illustrates the results for two patients who underwent STSG one year later due to skin defects (Figure $5 A, C$ ) in the palm. The NPWT scar surface (Figure $5 B$ ) was more regular than that of conventional mechanical fixation (Figure 5D). Figure 6 shows the results of two patients who underwent STSG one year later due to the skin defects (Figure $6 A, C$ ) in the opisthenar, with the scar surface after NPWT (Figure 6B) being more regular than that of conventional mechanical fixation (Figure 6D).

\section{Discussion}

For any skin graft surgery, graft fixation is particularly critical for graft survival, requiring appropriate pressure, stable tension, and adequate drainage. Conventionally, a tie-over bolster dressing and elastic bandage dressing are the most commonly used methods for securing skin grafts. Nevertheless, the use of bolster fixation requires experience and well-trained surgical skills to make the skin graft adhere closely to a recipient wound with an irregular surface. Surgical fixation with tie-over bolster and elastic bandage may lead to inappropriate tension and pressure, and their drainage effects are limited and not predictable. As a result, high pressure may lead to skin graft necrosis, whereas insufficient drainage may promote infection, both of which may impair graft survival. For some anatomical sites, such as the head, neck, hand and foot, the shape of the wound may be complex and irregular, and the pressure needs to be subtly adjusted. Unlike manual fixation techniques, NPWT applies negative pressure to the space between the skin graft and the recipient site: it removes space and pulls the entire skin graft with uniform pressure. Laboratory and clinical studies have shown that NPWT increases wound blood flow, oxygen concentration, and granulation tissue formation and decreases the accumulation of fluid and bacteria $(17,18)$. Several studies have demonstrated that NPWT can be used successfully for securing skin grafts, especially in exudative, irregular, or mobile recipient wounds and in complex anatomic sites (19-21). The advantages of NPWT over mechanical dressings include improved graft survival and decreased complications, mainly due to the removal of fluid beneath the graft, reliable and subtle pressure, and prevention of shear forces, consistent with our research results.

Several studies have shown that NPWT can improve the appearance of incisional scars (22-24), though the aesthetic effect of scarring skin grafts fixed by NPWT is not clear. In our study, a significant difference in the surface smoothness or relief of hand scars was observed. Although the mechanism is not clear, some animal experiments have shown that pressure can reduce the thickness of the scar dermis but has no significant effect on the thickness of the epidermis. The decrease in thickness may be due to the loss of local fluid, cells and/or the extracellular matrix (25). In the process of scar formation, the focal adhesion complex may play an important role in the mechanical signal transduction of pressure and affect the proliferation 

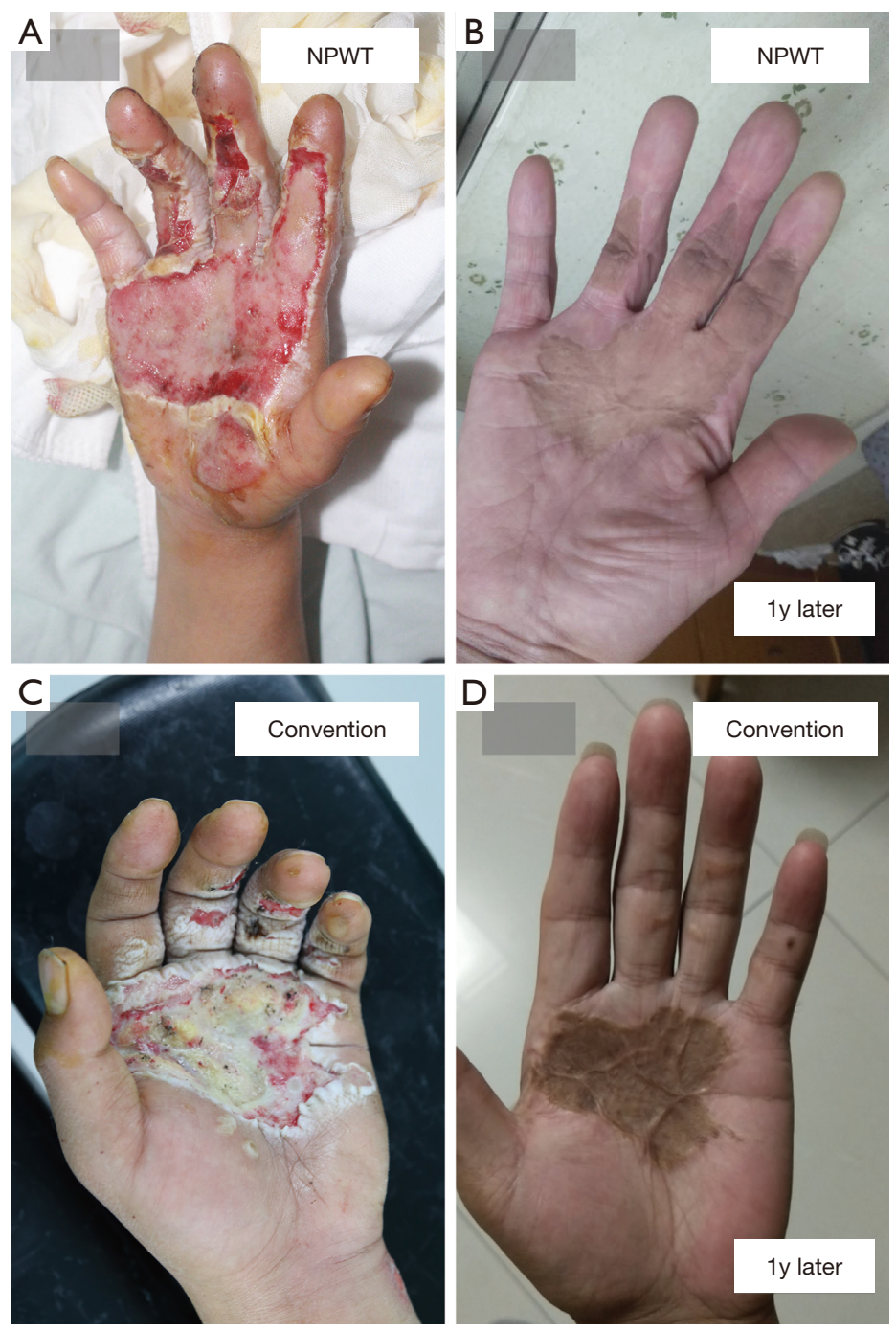

Figure 5 Comparison of long-term efficacy between negative pressure wound therapy (NPWT) and conventional mechanical fixation in palm. (A) Preoperatively; (B) appearance 1 year after the split-thickness skin graft fixed with NPWT; (C) preoperatively; (D) appearance 1 year after the split-thickness skin graft fixed with conventional mechanical fixation. NPWT, negative-pressure wound therapy.

and collagen synthesis of downstream fibroblasts (26). We speculate that as NPWT provides more uniform pressure than conventional mechanical fixation, the thickness of the scar dermis tends to be consistent, and the surface of the scar is flatter and more regular. Of course, further research is needed to confirm this hypothesis.

NPWT helps to maintain strong adhesion, even at sites where it is difficult to maintain steady pressure. In theory, this technique can be applied for any graft recipient site if there is sufficient space to place a film dressing securely around the site. Hand wounds are often irregular and complex, making it very difficult to fix a skin graft. At the same time, due to the special function and use of the hand in daily life, patients are often unable to maintain absolute fixation of all areas of the hand; thus, conventional mechanical fixation has difficulty in achieving continuous fixation and stable pressure of the skin graft and the surrounding skin. NPWT can provide continuous and stable pressure, with more obvious advantages for complex wounds such as the hand, including the total graft survival rate and the long-term scar effect.

To evaluate the quality of scars, we used two representative scar scoring methods: VSS and POSAS. The VSS, which was developed by Sullivan et al. in 

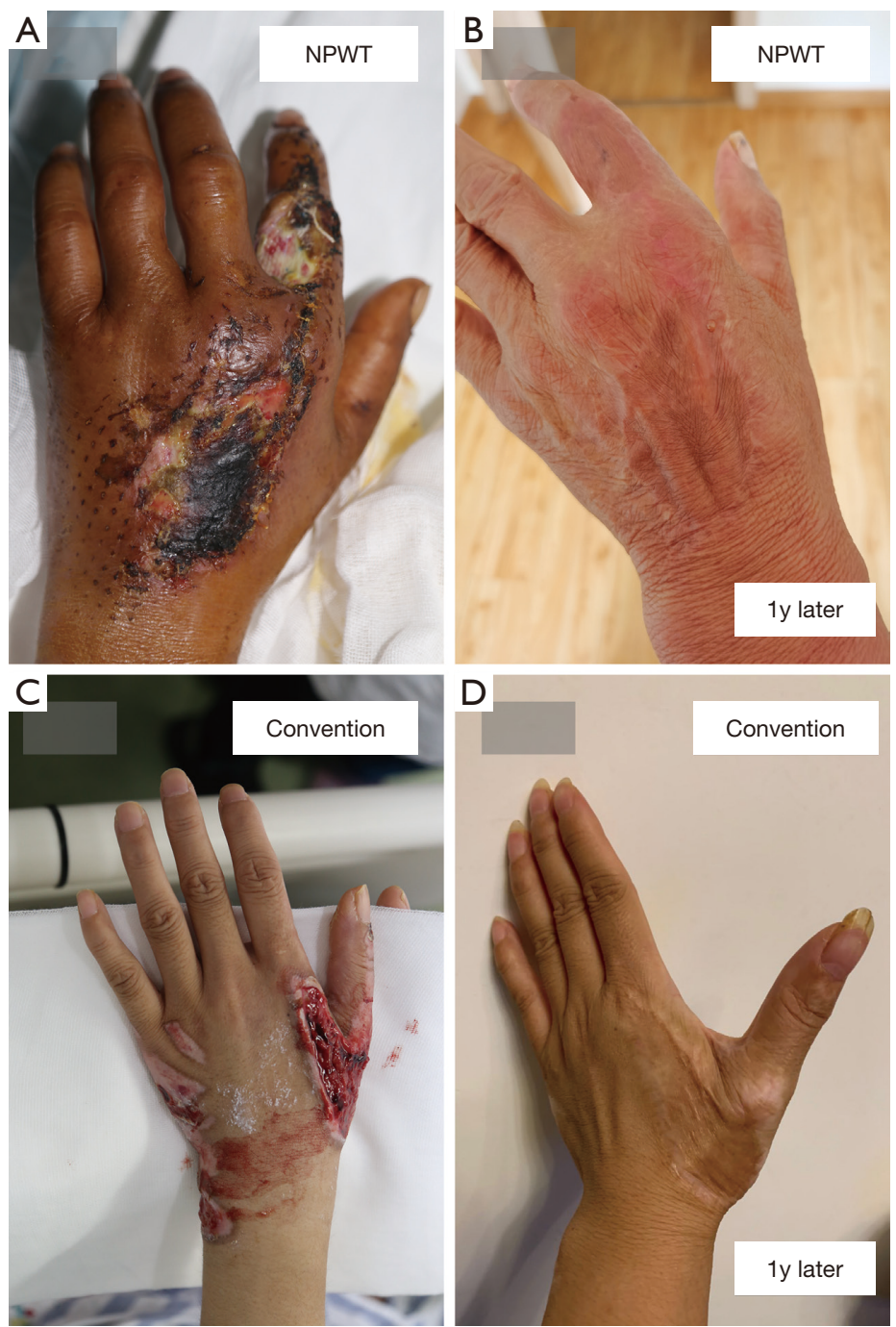

Figure 6 Comparison of long-term efficacy between negative pressure wound therapy (NPWT) and conventional mechanical fixation in opisthenar. (A) Preoperatively; (B) appearance 1 year after the split-thickness skin graft fixed with NPWT; (C) preoperatively; (D) appearance 1 year after the split-thickness skin graft fixed with conventional mechanical fixation. NPWT, negative-pressure wound therapy.

1990, calculates subscores in four categories (vascularity, pigmentation, pliability, and height of the scar) and aggregates the scores (27). The POSAS was developed in 2004 to emphasize the importance of subjective symptoms in patients, including pain and itching, while adding scar surface regularity and overall opinion scores for patients and observers. These factors were not considered in the previous scar assessment scale. Both are widely used scar assessment scales (28). The word "Pliability" in OSAS is equivalent to "Stiffness" in PSAS, as well as "Relief" and "Irregularity", which are different expressions for the observer and patient groups. In our study, the evaluation results of the four items of pigmentation, vascularization, pliability and height in the VSS were consistent with those in the POSA. Overall, the scar surface regularity according to the POSA indicated a difference with regard to the hands.

Our research has some limitations. First, because instruments that can accurately measure the area of irregular wounds have not been widely used, even in recent years, we adopted a method in which the qualitative analysis was judged by clinicians to evaluate the survival of skin grafts, which may cause a certain bias. Second, due to the 
limited number of cases, we did not conduct a comparative analysis of the same etiology at the same site; in theory, therefore, the comparison between the two groups was more convincing.

\section{Conclusions}

Appropriate fixation is critical for the survival of a skin graft. NPWT is an effective method of fixing skin grafts. Compared with conventional mechanical fixation, NPWT can significantly improve the survival rate of STSG and reduce the infection rate. In the long term, NPWT can also improve scar surface regularity in the hand, and the esthetic effect is more satisfactory to clinicians and patients.

\section{Acknowledgments}

Funding: This work was supported by the National Natural Science Foundation of China (grant number: 81800241), Postgraduate Research \& Practice Innovation Program of Jiangsu Province (SJCX20_0484) and the Fundamental Research Funds for the Central Universities (14380479).

\section{Footnote}

Reporting Checklist: The authors have completed the STROBE reporting checklist. Available at http://dx.doi. org/10.21037/apm-20-1806

Data Sharing Statement: Available at http://dx.doi. org/10.21037/apm-20-1806

Conflicts of Interest: All authors have completed the ICMJE uniform disclosure form (available at http://dx.doi. org/10.21037/apm-20-1806). The authors have no other conflicts of interest to declare.

Ethics Statement: The authors are accountable for all aspects of the work in ensuring that questions related to the accuracy or integrity of any part of the work are appropriately investigated and resolved. Written informed consent was obtained from the patient for publication of this study and any accompanying images. The study was conducted in accordance with the Declaration of Helsinki (as revised in 2013). The research scheme was approved by the Ethics Committee of Nanjing Drum Tower Hospital (2020109-01).
Open Access Statement: This is an Open Access article distributed in accordance with the Creative Commons Attribution-NonCommercial-NoDerivs 4.0 International License (CC BY-NC-ND 4.0), which permits the noncommercial replication and distribution of the article with the strict proviso that no changes or edits are made and the original work is properly cited (including links to both the formal publication through the relevant DOI and the license). See: https://creativecommons.org/licenses/by-nc-nd/4.0/.

\section{References}

1. Johnson TM, Ratner D, Nelson BR. Soft tissue reconstruction with skin grafting. J Am Acad Dermatol 1992;27:151-65.

2. Patton ML, Mullins RF, Smith D, et al. An open, prospective, randomized pilot investigation evaluating pain with the use of a soft silicone wound contact layer vs bridal veil and staples on split thickness skin grafts as a primary dressing. J Burn Care Res 2013;34:674-81.

3. Struk S, Correia N, Guenane Y, et al. Full-thickness skin grafts for lower leg defects coverage: Interest of postoperative immobilization. Ann Chir Plast Esthet 2018;63:229-33.

4. Sawada Y, Yotsuyanagi T, Ara M, et al. Experiences using silicone gel tie-over dressings following skin grafting. Burns 1990;16:353-7.

5. Kamolz LP, Andel H, Haslik W, et al. Use of subatmospheric pressure therapy to prevent burn wound progression in human: first experiences. Burns 2004;30:253-8.

6. Körber A, Franckson T, Grabbe S, et al. Vacuum Assisted Closure Device Improves the Take of Mesh Grafts in Chronic Leg Ulcer Patients. Dermatology 2008;216:250-6.

7. Kantak NA, Mistry R, Halvorson EG. A review of negative-pressure wound therapy in the management of burn wounds. Burns 2016;42:1623-33.

8. Chio EG, Agrawal A. A randomized, prospective, controlled study of forearm donor site healing when using a vacuum dressing. Otolaryngol Head Neck Surg 2010;142:174-8.

9. Ho MW, Rogers SN, Brown JS, et al. Prospective evaluation of a negative pressure dressing system in the management of the fibula free flap donor site: a comparative analysis. JAMA Otolaryngol Head Neck Surg 2013;139:1048-53. 
10. Schlosser KA, Otero J, Lincourt A, et al. Management of Surgical Incisions Using Incisional Negative-Pressure Therapy. Plast Reconstr Surg 2019;143:15s-20s.

11. Ehrl D, Heidekrueger PI, Broer PN, et al. Topical Negative Pressure Wound Therapy of Burned Hands: Functional Outcomes. J Burn Care Res 2018;39:121-8.

12. Perez D, Wildi S, Demartines N, et al. Prospective evaluation of vacuum-assisted closure in abdominal compartment syndrome and severe abdominal sepsis. J Am Coll Surg 2007;205:586-92.

13. Moisidis E, Heath T, Boorer C, et al. A prospective, blinded, randomized, controlled clinical trial of topical negative pressure use in skin grafting. Plast Reconstr Surg 2004;114:917-22.

14. Bloemen MC, van der Wal MB, Verhaegen PD, et al. Clinical effectiveness of dermal substitution in burns by topical negative pressure: a multicenter randomized controlled trial. Wound Repair Regen 2012;20:797-805.

15. Clark JM, Rychlik S, Harris J, et al. Donor site morbidity following radial forearm free flap reconstruction with split thickness skin grafts using negative pressure wound therapy. J Otolaryngol Head Neck Surg 2019;48:21.

16. Marsidi N, Boteva K, Vermeulen SAM, et al. To Tie or Not to Tie-Over Full-Thickness Skin Grafts in Dermatologic Surgery: A Systematic Review of the Literature. Dermatol Surg 2021;47:18-22.

17. Morykwas MJ, Argenta LC, Shelton-Brown EI, et al. Vacuum-assisted closure: a new method for wound control and treatment: animal studies and basic foundation. Ann Plast Surg 1997;38:553-62.

18. Armstrong DG, Lavery LA. Negative pressure wound therapy after partial diabetic foot amputation: a multicentre, randomised controlled trial. Lancet 2005;366:1704-10.

19. Molnar JA, DeFranzo AJ, Marks MW. Single-stage approach to skin grafting the exposed skull. Plast Reconstr
Surg 2000;105:174-7.

20. Schneider AM, Morykwas MJ, Argenta LC. A new and reliable method of securing skin grafts to the difficult recipient bed. Plast Reconstr Surg 1998;102:1195-8.

21. Hallberg H, Holmström H. Vaginal construction with skin grafts and vacuum-assisted closure. Scand J Plast Reconstr Surg Hand Surg 2003;37:97-101.

22. Tanaydin V, Beugels J, Andriessen A, et al. Correction to: Randomized Controlled Study Comparing Disposable Negative-Pressure Wound Therapy with Standard Care in Bilateral Breast Reduction Mammoplasty Evaluating Surgical Site Complications and Scar Quality. Aesthetic Plast Surg 2018;42:1176.

23. Tanaydin V, Beugels J, Andriessen A, et al. Randomized Controlled Study Comparing Disposable NegativePressure Wound Therapy with Standard Care in Bilateral Breast Reduction Mammoplasty Evaluating Surgical Site Complications and Scar Quality. Aesthetic Plast Surg 2018;42:927-35.

24. Nagata T, Miura K, Homma Y, et al. Comparison between Negative-Pressure Fixation and Film Dressing in Wound Management after Tissue Expansion: A Randomized Controlled Trial. Plast Reconstr Surg 2018;142:37-41.

25. Alkhalil A, Tejiram S, Travis TE, et al. A Translational Animal Model for Scar Compression Therapy Using an Automated Pressure Delivery System. Eplasty 2015;15:e29.

26. Rustad KC, Wong VW, Gurtner GC. The role of focal adhesion complexes in fibroblast mechanotransduction during scar formation. Differentiation 2013;86:87-91.

27. Sullivan T, Smith J, Kermode J, et al. Rating the burn scar. J Burn Care Rehabil 1990;11:256-60.

28. Draaijers LJ, Tempelman FR, Botman YA, et al. The patient and observer scar assessment scale: a reliable and feasible tool for scar evaluation. Plast Reconstr Surg 2004;113:1960-5; discussion 6-7.
Cite this article as: Mo R, Ma Z, Chen C, Meng X, Tan Q. Short- and long-term efficacy of negative-pressure wound therapy in split-thickness skin grafts: a retrospective study. Ann Palliat Med 2021;10(3):2935-2947. doi: 10.21037/apm-20-1806 